\title{
Fragmento bíblico en arameo cristiano palestinense procedente de la Qubbat al-Haznah (Damasco): Restitución de lecturas ilegibles y estudio de la versión (1 Sam 7,14-8,4-8)*
}

\author{
Juan Pedro Monferrer-Sala** \\ Universidad de Córdoba \\ ORCID ID: https://orcid.org/0000-0001-9979-1890
}

En este artículo ofrecemos un análisis del fragmento XVII ${ }^{\mathrm{rv}}$ (1 Sam 7,14-8,4-8) perteneciente al corpus de textos bíblicos en arameo cristiano palestinense con un doble objetivo: en primer lugar, demostrar que la versión fue realizada a partir de un texto griego de la familia de la LXX, en la que el traductor también utilizó una o más versiones siriacas; en segundo lugar, a partir de los datos obtenidos en el análisis anterior, hemos adoptado un criterio metodológico con el objeto de poder restaurar el texto fragmentario a base de restituir una serie de las lecturas ilegibles que exhibe el fragmento.

Palabras Clave: Traducción de la Biblia, arameo cristiano palestinense, LXX, restitución de lecturas ilegibles.

Biblical Fragment in Christian Palestinian Aramaic from Qubbat al-Hुaznah (Damascus): Restoration of Illegible Readings and Translation Study.- In this article we offer an analysis of fragment XVII-v $(1$ Sam 7:14-8.4-8) belonging to the corpus of biblical texts in Christian Palestinian Aramaic with a twofold aim: firstly, we will show that the version was translated from a Greek text of the LXX family, although the translator also used one or more Syriac versions; secondly, based on the data obtained in the previous analysis, we have adopted a methodological criterion in order to be able to restore the fragmentary text through the restoration of a series of illegible readings exhibited in the present fragment.

Keywords: Bible translation; Christian Palestinian Aramaic; LXX; Restoration of illegible readings.

* Este trabajo se enmarca en el Proyecto de Investigación PGC2018-096807-B-I00: 'Estudio y Edición de manuscritos bíblicos y patrísticos griegos, árabes y latinos', financiado por el Ministerio español de Ciencia, Innovación y Universidades.

**ff1mosaj@uco.es

Copyright: (C) 2021 CSIC. Este es un artículo de acceso abierto distribuido bajo los términos de la licencia de uso y distribución Creative Commons Reconocimiento 4.0 Internacional (CC BY 4.0). 


\section{INTRODUCCIÓN}

Aunque en los albores del siglo xx Bruno Violet diera cuenta al mundo académico occidental de algunos de los materiales que formaban parte de los fondos manuscritos conservados en la Qubbat al-Haznah de la Mezquita de los Omeyas en Damasco ${ }^{1}$, los especialistas no han prestado excesivo interés a la importancia de los materiales -judíos, cristianos e islámicos- que allí se preservaron en varias lenguas, llegando incluso a subestimar el descubrimiento ${ }^{2}$. La importancia de estos textos -que conocerían un indeseable periplo a tierras germanas, para luego volver a Damasco y Turquía, acabando algunos de ellos en paradero desconocido en la actualidad- es variada en función del interés que por los materiales tenga cada investigador, obviamente. Pero, además, lo que es cierto es que en conjunto esos textos son la herencia de una realidad sociolingüística en la que dos elementos, multilingüismo y multiculturalismo, desempeñaron un papel de primer orden a nivel cultural, como de ello dan fe los textos preservados ${ }^{3}$.

El corpus de las versiones bíblicas generadas por una comunidad melkita palestinense constituye una parte importante de la literatura eclesiástica vertida, y compuesta, en un dialecto cercano al arameo judeo palestinense, el arameo cristiano palestinense $(\mathrm{ACP})^{4}$, que como sucede con la versión

${ }^{1}$ Cordula Bandt y Arndt Rattmann, «Die Damaskusreise Bruno Violets 1900/1901 zur Erforschung der Qubbet el-Chazne», Codices Manuscripti 76/77 (2011) págs. 1-20. $C f$. D. Hermann Freiherr von Soden, «Bericht über die in der Kubbet in Damaskus gefundenen Handschrifttenfragmente», Sitzungsberichte der Königlich Preußischen Akademie der Wissenschaften. Philosophisch-historische Classe (1903) Halbband II, págs. 825-883.

2 Paolo Radiciotti y Arianna D’Ottone, «I frammenti della Qubba al-Hazna di Damasco. A propósito di una scoperta sottovaluata», Nea Roma 5 (2008) págs. 45-74.

3 Arianna D'Otтone RAmBach, «Manuscripts as Mirrors of a Multilingual and Multicultural Society: The Case of the Damascus Find», en Negotiating Co-Existence: Communities, Cultures and Convivencia in Byzantine Society, ed. Barbara Crostini y Sergio La PorTa (Trier: Wissenschaftlicher Verlag, 2013) págs. 63-88.

${ }^{4}$ Acerca de este registro, véanse Theodor NÖLDEKE, «Über den christlich-palästinischen Dialekt», Zeitschrift der deutschen morgenländischen Gesellschaft XXII (1868) págs. 443-527; Christa Müller-Kessler, Grammatik des Christlich-Palästinisch-Aramäischen. I. Schriftlehre, Lautlehre, Formenlehre (Hildesheim-Zürich-New York: Gregor Olms, 1991) págs. 1-8 y Friedrich Schulthess, Grammatik des Christlich-Palästinischen Aramäisch, ed. Enno LitTMAnN, con notas de Theodor Nöldeke y el editor 
syro-hexaplar traducida por Pablo de Tellā en el siglo vII ${ }^{5}$, la syro-luciánica del siglo VI $^{6}$ y la de Jaime de Edesa ${ }^{7}$, de comienzos del siglo VIII, se las supone realizadas a partir de un texto griego ${ }^{8}$ de la familia de la $\mathrm{LXX}^{9}$. Sin que nos opongamos a la afirmación precedente, en cuyos orígenes habrá que tener en consideración la práctica traductora oral del griego al arameo realizada por intérpretes ${ }^{10}$, que en líneas generales es correcta, sin embargo en este trabajo nos reafirmamos en nuestra teoría de que el o los traductores

(Tübingen: J.C.B. Mohr, 1924; reimp. Hildesheim: Georg Olms, 1965) págs. 1-4. También Klaus BeYer, The Aramaic Language: Its Distribution and Subdivisions. Translated from the German by John F. Healey (Göttingen: Vandenhoeck \& Ruprecht, 1986) págs. 51-53, cf. pág. 36 y Christa Müller-Kessler, «Christian Palestinian Aramaic and Its Significance to the Western Aramaic Dialect Group», Journal of the American Oriental Society 119:4 (1999) págs. 631-636.

5 Timothy M. LAw, «La version syro-hexaplaire et la transmission textuelle de la Bible grecque», en L'Ancien Testament en syriaque, ed. Françoise BriQuel-Chatonnet y Phillippe Le Moigne (=Études syriaques 5 [Paris: Geuthner, 2008]) págs. 101-120.

${ }^{6}$ Sebastian P. BROCK, «Les versions syriaques de l'Ancien Testament: quelques approches récentes», en L'Ancien Testament en syriaque, págs. 21-32: 27.

7 Alison G. SAlvesen, «La version de Jacques d'Edesse», en L'Ancien Testament en syriaque, págs. 121-139.

8 Rubens Duval, Anciennes littératures chrétiennes. II. La literature syriaque (Paris: Librairie Victor Lecoffre, J. Gabalda \& C Cie, 1907) págs. 43-48. Cf. Christa MüllerKessller, «An Overlooked Christian Palestinian Aramaic Witness of the "Dormition of Mary" in Codex Climaci Rescriptus (CCR IV)», Collectanea Christiana Orientalia 16 (2019) págs. 81-98: 86.

9 Véanse al respecto Henry Barclay SwETE, An Inroduction to the Old Testament in Greek. Revised by Richard Rusden Ottley. With an Appendix containing the Letter of Aristeas, ed. H. St. J. Thackeray (Cambridge: Cambridge University Press, 1900) y Natalio Fernández Marcos, The Septuagint in Context: Introduction to the Greek Version of the Bible. Trad. Wilfred G.E. Watson (Leiden-Boston-Colonia: Brill, 2000). Para el texto de 1-2 Samuel, véanse Sidney Jellicoe, The Septuagint and Modern Study (Oxford: Clarendon Press, 1968) págs. 283-290 (junto con 1-2 Reinos). Véase, además, la bibliografía clasificada por Sebastian P. Brock, Charles T. Fritsch y Sidney Jellicoe, A Classified Bibliography of the Septuagint (=Arbeiten zur Literatur und Geschichte des Hellenistischen Judentums VI [Leiden: E. J. Brill, 1973]) págs. 105-107 y Cécile DognIEZ, Bibliography of the Septuagint/Bibliographie de la Septanta 1970-1993. Avec un préface de Pierre-Maurice Bogaert (=Supplements to Vetus Testamentum LX [Leiden: E. J. Brill, 1995]) págs. 156-163.

10 Sebastian P. Brock, «The Palestinian Syriac Version», en Bruce M. Metzger, The Early Versions of the New Testament: Their Origin, Transmission, and Limitations (Oxford: Clarendon Press, 1977) pág. 77, y BEYER, The Aramaic Language, pág. 52. 
de estos textos bíblicos, ciertamente utilizaron como texto base un texto griego de la familia de la LXX, pero además se sirvieron de otras versiones, especialmente siriacas, con las que revisaron y perfilaron su traducción, todo ello sin menoscabo de las aportaciones genuinas debidas al traductor de cada texto. Esta práctica de revisión o cotejo de la traducción, además, no tuvo por qué realizarse una vez acabada la traducción, sino que pudo darse, incluso, durante el mismo proceso de revisión, como sucede en otros casos. Sea como fuere, lo que es evidente es que esa práctica de cotejo, ya final ya en pleno proceso traductor, más que una práctica es de hecho una estrategia de traducción característica, aunque en modo alguno exclusiva ${ }^{11}$, de los traductores melkitas bilingües o trilingües greco-(arameo-)árabes posteriores, sin duda heredada de las escuelas de traductores anteriores ${ }^{12}$.

Que las versiones bíblicas en ACP son el resultado de la utilización de más de un texto en el proceso de composición traductora es una cuestión que nos parece evidente. Así hemos creído demostrarlo con anterioridad ${ }^{13}$, pero ahora reforzamos nuestra hipótesis allí adelantada a modo de praeparatio con el estudio de este nuevo texto, que en algunos de sus versículos exhibe una serie de rasgos e interesantes evidencias con respecto tanto a las versiones utilizadas, como a las estrategias propias adoptadas en la traducción.

En este caso, el fragmento preservado contiene, con carácter general, una traducción verbum e verbo del texto base griego utilizado en la tra-

11 Alison SAlvesen, The Books of Samuel in the Syriac Version of Jacob of Edessa (=Monographs of the Peshitta Institute Leiden 10 [Leiden-Boston-Köln: Brill, 1999]) págs. 90 (texto siriaco) y 67 (trad. inglesa), $c f$. además págs. ix-xi.

12 Véanse en esta línea Mt. Sinai Arabic Codex 151, I. Pauline Epistles, ed. Harvey StaAl (Louvain: CorpusSCO, 1983) págs. 40 (n. 24), 45 (n. 14), 138 (n. 8) y 145 (n. 8); y Juan Pedro Monferrer-SAlA, «An early Fragmentary Christian Palestinian Rendition of the Gospels into Arabic from Mār Sābā (MS Vat. Ar. 13, 9th c.)», Intellectual History of the Islamicate World 1 (2013) págs. 69-113: 71, 73 y 95-96; «The Pauline Epistle to Philemon from Codex Vatican Arabic 13 (Ninth Century CE). Transcription and Study», Journal of Semitic Studies 60:2 (2015) págs. 341-371: 359 y 368, y «Translating the Gospels into Arabic from Syriac: Vatican Arabic 13 Restored Section, Strategies and Goals», Arabica 62 (2015) págs. 435-458: 437 y 456.

13 Juan Pedro Monferrer-Sala, «Dos fragmentos en arameo cristiano-palestinense del libro del Génesis procedentes de la Mezquita de los Omeyas de Damasco», Sefarad 77:1 (2017) págs 65-90: 72-90. 
ducción ${ }^{14}$, una técnica que los traductores cristianos de lengua siriaca conocían y dominaban a la perfección ${ }^{15}$. Sin embargo, en casos puntuales, la versión ofrece lecturas que corresponden a otras versiones, siriacas, y en los menos de los casos el texto exhibe lo que podemos calificar como 'variantes de traducción', como consecuencia de una determinada estrategia adoptada por el traductor del texto, por lo general modulaciones en diverso grado, que a su vez devienen en cambios exegéticos, acomodaciones contextuales o clarificaciones del original utilizado ${ }^{16}$.

Esta práctica de utilizar varias versiones, griegas y siriacas, no es en modo alguno extraña entre los traductores melkitas, pues de hecho seguirán utilizándola una vez se imponga el árabe como nueva lingua franca en el Oriens Christianus y estos tengan que trasvasar al árabe las Sagradas Escrituras, entre una ingente cantidad de materiales de géneros y tipologías diversas.

En este trabajo, además del estudio de la traducción, formulamos una serie de hipótesis con las que restituimos lecturas del fragmento que resultaron ilegibles para los editores anteriores. Estas restituciones de lecturas perdidas que hemos incorporado en la edición son, asimismo, reproducidas en transliteración en el estudio que sigue a la edición.

\section{NuEVA EDICIÓN DEL FRAGMENTO XVII ${ }^{\mathrm{R}-\mathrm{v}}$ CON LECTURAS RESTITUIDAS}

El texto conservado del fragmento objeto de estudio contiene lecturas fragmentarias de 1 Sam 7,14-8,4 y 1 Sam 8,4-8, identificados respectivamente como Frag. XVIIr y Frag. XVIIv. El fragmento ha sido editado por Müller-Kessler y Sokoloff ${ }^{17}$. Sin embargo, como sucede con otros textos, no se trata de la primera edición, pues ya con anterioridad los editaron

14 Acerca del literalismo en las versiones bíblicas, véase James BARR, The Typology of Literalism in ancient biblical translations (Göttingen: Vandenhoeck \& Ruprecht, 1979).

15 Sebastian P. BROCK, «Aspects of Translation Technique in Antiquity», Greek, Roman and Byzantine Studies 20:1 (1979) págs. 69-87.

16 Véanse al respecto los ejemplos que estudia Janet W. DYK, Language System, Translation Technique, and Textual Tradition in the Peshitta of Kings (=Monographs of the Peshitta Institute Leiden 19 [Leiden-Boston: Brill, 2013]) págs. 47-77.

17 Cf. A Corpus of Christian Palestinian Aramaic. Volume I: The Christian Palestinian Aramaic Old Testament and Apocrypha Version from the Early Period, ed. Christa MüLLERKessLer y Michael SoKoloff (Groningen: Styx Publications, 1997) págs. 97-98 y 222. 
primero Schulthess ${ }^{18}$ y posteriormente Goshen-Gottstein ${ }^{19}$. Ambos fragmentos proceden de Damasco, aunque se desconoce su actual localización, como sucede con otros veintiún fragmentos ${ }^{20}$, a dos de los cuales dedicamos anteriormente un estudio en esta misma revista ${ }^{21}$.

Signos utilizados

$\begin{array}{ll}{[\ldots]} & \text { Vacat } \\ {[\mathrm{xxx}]} & \text { grafema o secuencia añadida en la edición } \\ { }^{\mathrm{X}}{ }^{\urcorner} & \text {caracter(es) parcialmente legibles } \\ { }_{\mid} \mathrm{Xxx}_{\mid} \mid & \text {restitución de lecturas ilegibles }\end{array}$

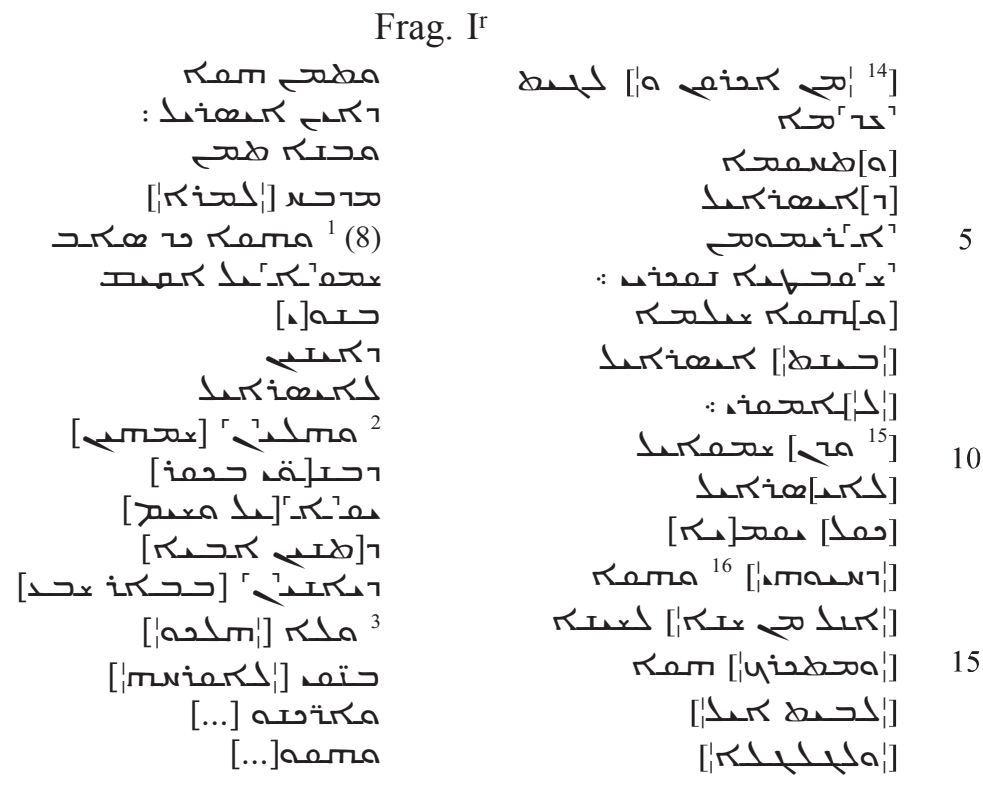

18 Friedrich Schulthess, Christlich-palästinische Fragmente aus der OmajjadenMoschee zu Damaskus (Berlin, 1905) págs. 31-32.

19 Moshe Goshen-Gottstein, The Bible in the Syropalestinian Version, Part I: Pentateuch and Prophets. With the assistance of H. SHIRUN (=Publications of the Hebrew University Bible Project Monograph Series 4 [Jerusalem: Magnes Press-The Hebrew University, 1973]) pág. 53.

20 A Corpus of Christian Palestinian Aramaic. Volume I, ed. MüLler-KessLer y Sokoloff, pág. 232.

${ }_{21}$ Monferrer-Sala, «Dos fragmentos en arameo cristiano-palestinense del libro del Génesis». 


$$
\begin{aligned}
& \text { אسمن] [... }
\end{aligned}
$$

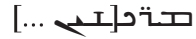

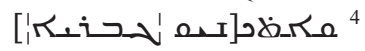

$$
\begin{aligned}
& \text { [LRines]గר }
\end{aligned}
$$

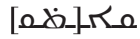

Frag. I

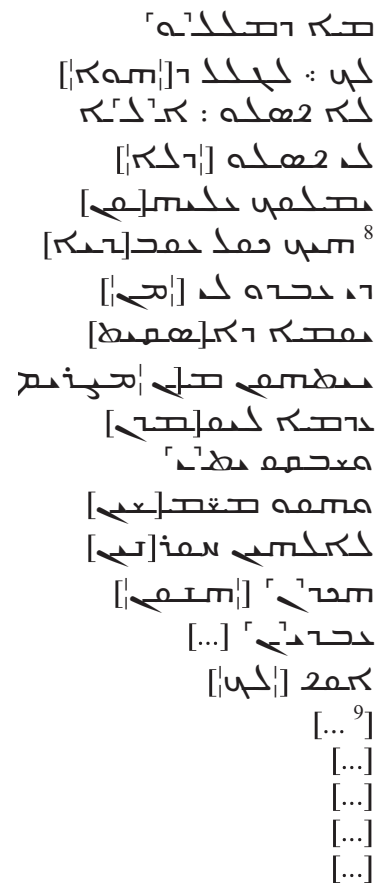

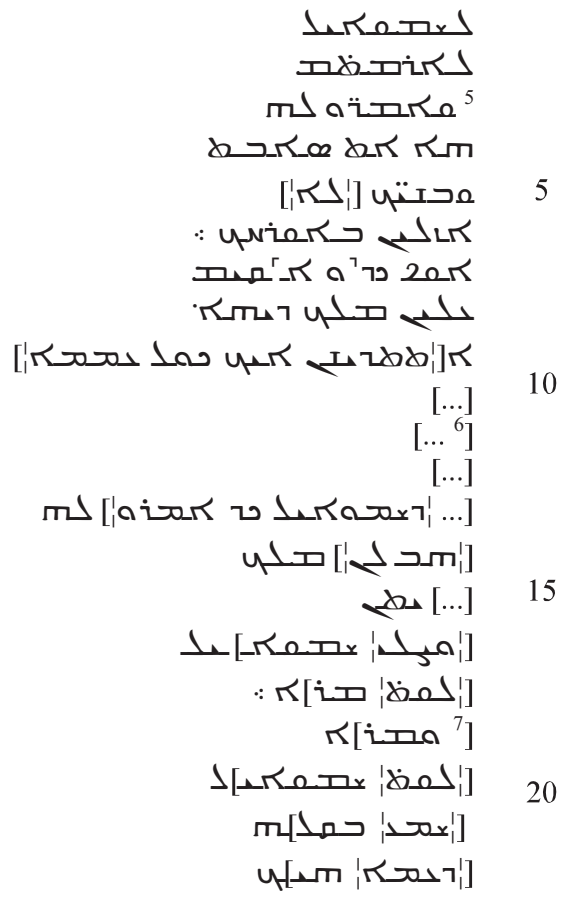

\section{ANÁlisis DE LOS FRAGMENTOS}

Para el análisis de estos dos fragmentos en ACP nos hemos servido del texto griego de la LXX editado por Fernández Marcos y Busto Sáiz ${ }^{22}$, cuya lectura sustituimos solo en casos muy concretos por no

22 Natalio Fernández Marcos y José Ramón Busto Sáiz, El texto antioqueno de la

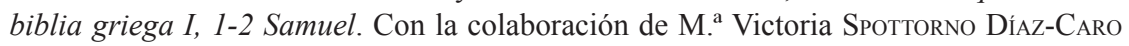


representar, en nuestra opinión, la lectura seguida por $\mathrm{ACP}^{23}$; asimismo, hemos cotejado la versión de ACP con el texto siriaco de la Pešitțā ${ }^{24}$, la versión siriaca de Jacobo de Edesa ${ }^{25}$ y los scholia al AT de Barhebreo ${ }^{26}$, además de ofrecer referencias al texto hebreo masorético, con el objeto de poder valorar la naturaleza de los dos fragmentos. En el caso de la Vulgata utilizamos la edición de Fischer ${ }^{27}$.

En cuadros sinópticos ofrecemos los textos confrontados de la LXX y de ACP, acompañados de una traducción lo más literal posible de la LXX en la parte inferior ${ }^{28}$, incluyendo entre paréntesis aquellas palabras o sintagmas ausentes en ACP. Señalamos en cada lugar las restituciones de términos que hemos indicado en la nueva edición que ofrecemos. En el cuerpo del análisis, cuando lo estimamos pertinente, damos la traducción de ACP por términos, sintagmas u oraciones en su caso, al tiempo que incorporamos otras versiones con el fin de obtener mayor información con la que valorar las estrategias de traducción que exhibe el texto ACP.

Abreviaturas y signos utilizados

ACP Texto(s) en arameo cristiano palestinense

$\mathrm{B}^{\mathrm{s}} \quad$ Scholia de Barhebreo sobre el AT

LXX Septuaginta

Syr ${ }^{\mathrm{je}} \quad$ Versión siriaca de Jacobo de Edesa

y S. Peter Cowe (=Textos y Estudios «Cardenal Cisneros» de la Biblia Políglota Matritense 50 [Madrid: CSIC, 1989]) págs. 21-22.

23 The Old Testament in Greek II/1, ed. Alan England Brook, Norman McLean y Henry St. John Thackeray (Cambridge: Cambridge University Press, 1927).

24 The Old Testament in Syriac according to the Peshitta Version, Part II, fascicle 2. Judges - Samuel, ed. Pieter Arie Hendrik DE BoER (Leiden: E.J. Brill, 1978).

25 SALVESEn, The Books of Samuel in the Syriac Version of Jacob of Edessa, págs. 22-24.

${ }^{26}$ Barhebraeus' Scholia on the Old Testament. Part I: Genesis-II Samuel, ed. Martin Sprengling (=The University of Chicago Oriental Institute Publications XIII [Chicago, IL: The University of Chicago Press, 1931]) págs. 305-306.

27 Biblia sacra iuxta Vulgatam versionem, ed. Bonifatius Fischer et al., preparada por Roger Gryson (Stuttgart: Deutsche Bibelgesellschaft, 1994: reimp. de la ed. 1969).

${ }^{28}$ Para la traducción de estos pasajes, de Natalio Fernández MARCos, véase la versión incluida en Natalio Fernández Marcos y M. ${ }^{\text {a }}$ Victoria Spottorno Díaz-CARo (coords.), La Biblia griega Septuaginta. II. Libros históricos (Salamanca: Sígueme, 2011) págs. 213-214. 


$\begin{array}{ll}\text { Syr }^{\mathrm{p}} & \text { Pešițtā } \\ \mathrm{TH} & \text { Texto hebreo } \\ \mathrm{Vg} & \text { Vulgata } \\ {[\ldots]} & \text { Vacat } \\ {[\mathrm{xxx}]} & \text { grafema o secuencia añadida en la edición } \\ { }^{\mathrm{X}}{ }^{\top} & \text { caracter(es) parcialmente legibles } \\ { }_{\mid \mathrm{Xxx}_{\mid}} & \text {Restitución de lecturas ilegibles } \\ (\ldots) & \text { texto omitido en la traducción } \\ (\mathrm{xxx}) & \text { texto de la LXX ausente en ACP }\end{array}$

A) Frag. XVIIr

$$
7,14^{29}
$$

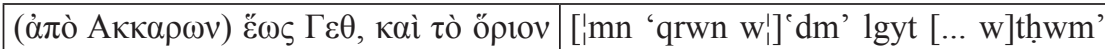

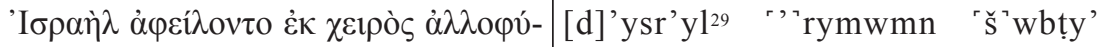

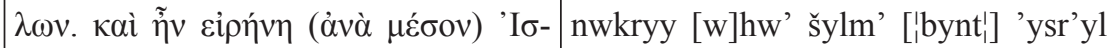

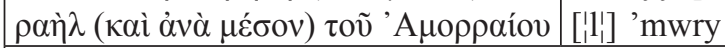

(desde Akkarón) hasta Get; y el límite de Israel quitaron de mano de los extranjeros; y hubo paz (en medio de) Israel (y en medio del) amorreo

El sintagma preposicional 'dm' lgyt ('hasta Geth') es traducción literal

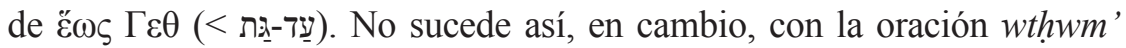
d'ysr'yl 'rymwmn šwbty' nwkryy ('la frontera de Israel fue arrebatada a la tribu de los extranjeros'), en la que el término šwbty' ('tribu') no traduce

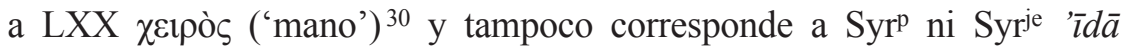
('mano', cf. '̄idā dapleštoye, 'la mano de los filisteos' < tal vez sea una modulación de àं ò $\alpha \lambda \lambda o \varphi v ́ \lambda \omega v$ ('de los extranjeros') ${ }^{31}$. El plural nwkryy es la traducción de LXX $\dot{\alpha} \lambda \lambda o \varphi v ́ \lambda \omega v^{32}$. voz empleada en la LXX (y en una ocasión en Hch) ${ }^{33}$ a partir del libro de los Jueces en ade-

${ }^{29}[d]$ 'ysr' $y l$ es lectura propuesta por Gortein, cf. A Corpus of Christian Palestinian Aramaic. Volume I, ed. Müller-Kessler y Sokoloff, pág. 97, n. 1.

${ }^{30}$ Cf. Fernández Marcos y Busto Sáiz, El texto antioqueno de la biblia griega I, pág. 21 ad locum.

${ }^{31}$ Cf. The Old Testament in Greek II/1, ed. Brook, McLean, Thackeray, pág. 22 ad locum.

32 Takamitsu MuraoKa, A Greek-English Lexicon of the Septuagint (LouvainParis-Walpole, MA: Peeters, 2009) pág. 29a.

33 Robert Morgenthaler, Statistik des neutestamentlichen Wortschatzes (Frankfurt am Main: Götthelf-Verlag Zürich, 1958) pág. 71. 
lante en 269 ocasiones para verter el hebreo ${ }^{34}$ Delante del sustantivo enfático thwwm' ('la frontera') habría que restituir, como de hecho hacemos en el texto de la edición, la conjunción ' $\mathrm{y}$ ' ( $w$-). Por su parte, la oración $w h w$ ' šylm'... 'ysr'yl ('y hubo paz ... Israel') se corresponde

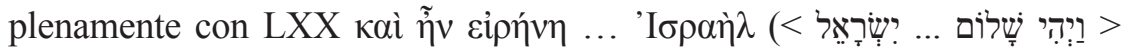
Syr ${ }^{\mathrm{p}}$ wahwō šlomō ... 'Isrāyel). En cuanto al último término preservado en este versículo, 'mwry ('el amorreo'), coincide con LXX ( 'A הָאוּמרי con el plural 'amūroyē ('los amorreos'). En cuanto a las restituciones que puedan llevarse a cabo en este versículo tenemos las tres siguientes: en el primer vacat, la lectura que proporcionan algunos manuscritos de la LXX

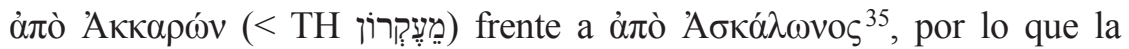
lacuna cabe ser restituida por $m n$ 'qrwn ('desde Akkarón'), añadiendo además la conjunción copulativa $w$-, que debe ir delante de ' $d m$ ', como así lo indican $\mathrm{Sr}^{\mathrm{p}}$ y Syr ${ }^{\mathrm{j}}$, que leen men 'Aqrūn wa'damō l-Gath. Para los

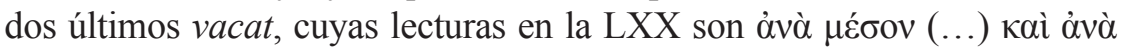

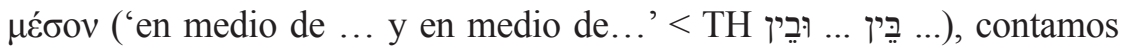
con las restituciones bynt ... $l$-, en cuyo favor habla la lectio que proporciona Syr ${ }^{\mathrm{j}}$ : baynot ... l-.

\section{7,15}

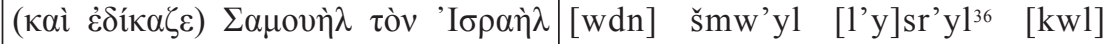

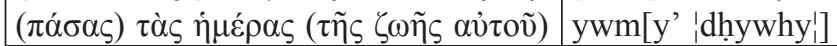

(Y juzgaba) Samuel a Israel (todos) los días de su vida

34 Roland DE VAUX, «Les philistins dans la Septante», en Forschung zur Bible: Wort, Lied und Gottesspruch. 1. Beiträge zur Septuaginta. Festschrift für Joseph Ziegler, ed. Josef SchreIner (Stuttgart: Echter Verlag Katholisches Biblewerk, 1972) págs. 185-194. Cf. Xavier JACQues, Index des mots apparentés dans la Septante (=Subsidia Biblica 1 [Roma: Biblical Institute Press, 1972]) pág. 12.

35 Fernández Marcos y Busto Sáiz, El texto antioqueno de la biblia griega I, pág. 21 ad locum. Cf. The Old Testament in Greek II/1, ed. Brook, McLean, Thackeray, pág. 22 ad locum; Fridericus FIELD, Origenis Hexaplorum quae supersunt; sive veterum interpretum graecorum in totum Vetus Testamentum fragmenta. I: Prolegomena, Genesis - Esther (Oxford: E Typographeo Clarendoniano, 1875) pág. 498.

$36[l ' y] s r$ 'yl es lectura propuesta por GoIteIn, $c f$. A Corpus of Christian Palestinian Aramaic. Volume I, ed. Müller-Kessler y SoKoloff, pág. 97 (n. 2). 
Las secuencias conservadas de este versículo son más escasas, concretamente tres, y coinciden plenamente tanto con la LXX como con la versión de Syr ${ }^{p}$, como indicamos en las correspondencias que enumeramos a continuación; Syr ${ }^{\mathrm{je}}$ ofrece una mínima amplificación en el primer segmento con respecto a la LXX y Syrp.

ACP LXX $\quad \operatorname{Syr}^{\mathrm{p}} \quad \mathrm{Syr}^{\mathrm{j}}$

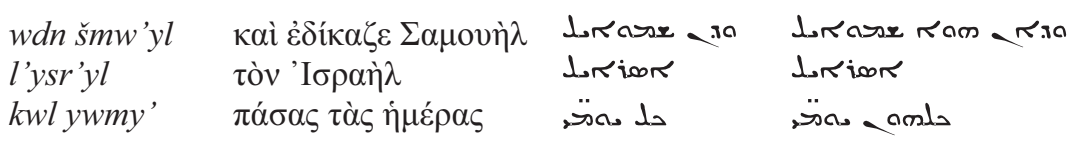

Como así hemos hecho en la edición, con alta probabilidad pueden ser restauradas las lectiones $w d n$ ('y juzgaba'), al comienzo del versículo, y $k w l$ ('todos') delante de ywmy' ('los días'). De las dos, la primera tal vez pudiera entrañar mayores obstáculos, pero la lectura que proporciona el texto de Syr ${ }^{\mathrm{p}}$, que es la que con mayor índice de frecuencia se utiliza para verter la

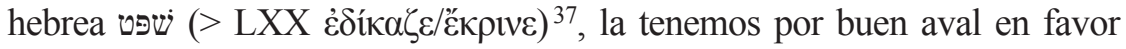
de la restitución ( $c f$. infra 7,17 d'yn 'ysryl). En cualquier caso, las lecturas proporcionadas por el texto fragmentario de ACP coinciden totalmente tanto con LXX como con Syrp, lo que hace realmente difícil saber si la versión del versículo completo fue realizada a partir de la LXX exclusivamente o si además utilizó, o incluso introdujo, nuevos elementos fruto de la estrategia adoptada por el traductor. En materia de restituciones, la lectura de la

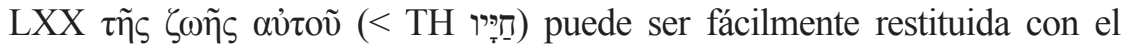
sintagma dhywhy ('de su vida'), que también recogen Syr (hayawhì) y Syr ${ }^{\mathrm{j} e}$ (dhayawhî), la primera de las dos sin el pronombre relativo.

7,16

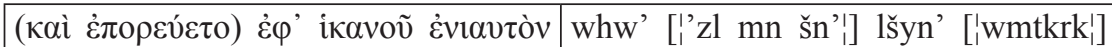

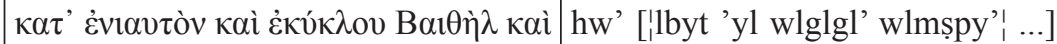

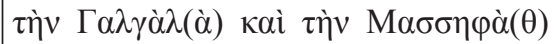

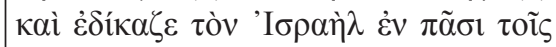

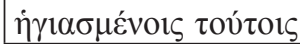

(E iba) cada año (y daba un rodeo por Betel, Galgal y Masefá, y juzgaba a Israel en todos estos (lugares) santificados)

37 Cf. Fernández Marcos y Busto Sárz, El texto antioqueno de la biblia griega I, pág. 21 ad locum.

SEFARAD, vol. 81:1, enero-junio 2021, págs. 21-42. ISSN: 0037-0894. https://doi.org/10.3989/sefarad.021-002 
En este versículo nos encontramos, si cabe, con mayores obstáculos aún que con el caso anterior, pues los términos preservados en el texto $\mathrm{ACP}$ poseen una menor relevancia en términos comparativos. De las tres secuencias conservadas, el sintagma lšyn' ('a año') corresponde a la

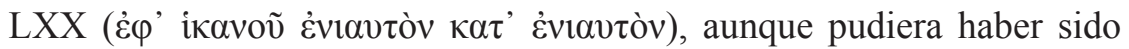
influida por la lectura atestiguada por Syrp men šnō lšno ('de año a año'),

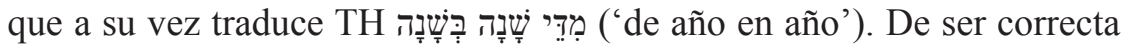
nuestra suposición, habría que restaurar en consecuencia el primer vacat con la lectura $m n \check{s} n$ ' ('de año...'), pero además precedida de la cons-

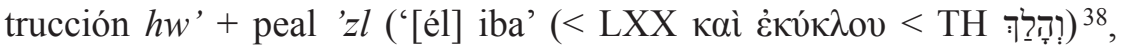
como así lo indican las versiones de Syr y Syr ${ }^{\mathrm{j} e}$ : wezal hwō men šnō lšnō. Del mismo modo, el segundo vacat es también susceptible de ser restituido por la forma participial precedida por conjunción copulativa wmtkrk ('y daba un rodeo'), como también recogen Syr ${ }^{\mathrm{p}}$ y Syr ${ }^{\mathrm{je}}$ al leer metkrek. El tercer vacat, aunque susceptible de restitución, al representar un segmento consonántico más amplio preferimos dejarlo sin restituir por los problemas que entraña, si exceptuamos los tres topónimos que van al principio de ese vacat. Para esos tres topónimos la LXX da la

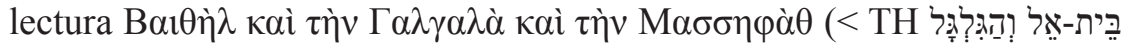

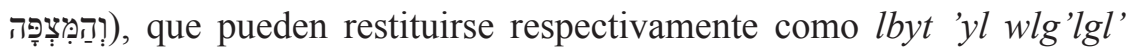
wlmsspy', la misma que ofrecen $\mathrm{Syr}^{\mathrm{p}}$ y $\mathrm{Syr}^{\mathrm{je}}$ (lbēt '̄il walgalgolō walmasfyō).

7,17

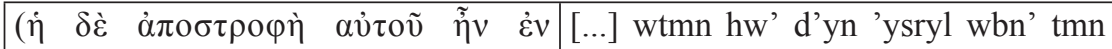

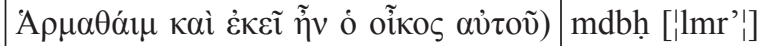

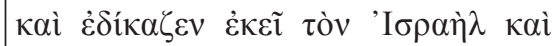

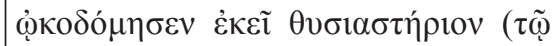
Kvpí( )

(Y su retorno a Ramatá, porque allí estaba su casa;) y juzgaba allí a Israel; y construyó allí un altar (al Señor)

38 Sobre la traducción de formas imperfectas de indicativo en ACP, véase Tarsee LI, Greek Indicative Verbs in the Christian Palestinian Aramaic Gospels: Translation Technique and the Aramaic Verbal System (=Perspectives on Linguistics and Ancient Languages 3 [Piscataway, NJ: Gorgias Press, 2013]) pág. 15 y ss. 
Las dos oraciones conservadas de este versículo en ACP nos permiten suministrar algún dato más de interés más para nuestro propósito de valoración final de la versión ACP. La primera de las dos oraciones, wtmn $h w$ ' d'yn 'ysryl ('y allí él juzgaba a Israel') no coincide totalmente

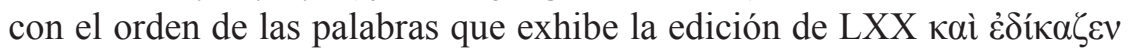

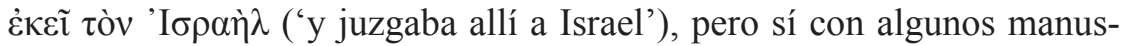

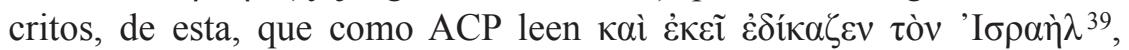
como recoge $\operatorname{Syr}^{\mathrm{p}}$ que lee wtaman da'en hwō l'İsrāyel, a partir de $\mathrm{TH}$

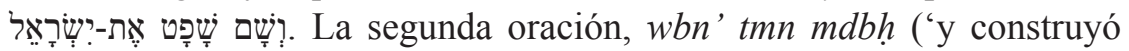

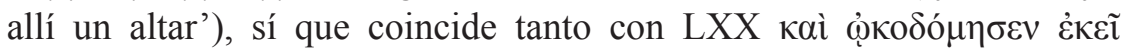

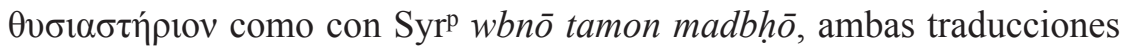

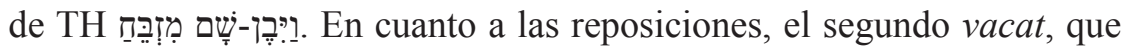

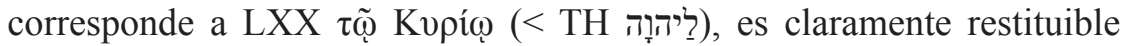
con el sintagma lmr' ('al Señor'), lectura atestiguada por $\operatorname{Syr}^{\mathrm{p}}$ y Syr ${ }^{\mathrm{je}}$ (l-Moryō). En el caso del primero, por su parte, dado el número de voces ilegibles, resulta harto arriesgado sugerir posibles lecturas con las que restituir el texto, máxime cuando las lecturas que dan $S_{y r}{ }^{p}$ y Syr ${ }^{\mathrm{je}}$ no coinciden plenamente.

\section{8,1}

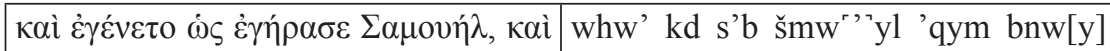

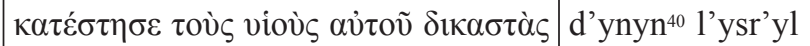
$\tau \tilde{\omega}{ }^{\prime} \mathrm{I} \sigma \rho \alpha \eta ் \lambda \lambda$

Y aconteció, cuando envejeció Samuel, que constituyó a sus hijos jueces a Israel

En este caso el versículo se nos ha conservado completamente en

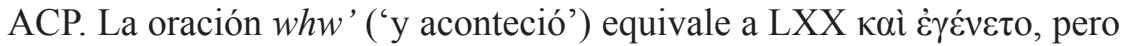
al propio tiempo esta lectio que exhibe ACP es la misma que ofrece Syrp whwō. Tanto la LXX como Syr ${ }^{\mathrm{p}}$ son traducción de TH וּיְ. La subordinada adverbial que depende de la oración principal anterior, $k d s^{\prime} b$ šmw'yl ('cuando envejeció Samuel'), también coincide con la versión de

39 The Old Testament in Greek II/1, ed. Brook, McLean, Thackeray, pág. 23 ad locum.

40 Ms. sic, cf. A Corpus of Christian Palestinian Aramaic. Volume I, ed. MüLlerKessler y SoKoloff, pág. 97 (n. 3).

SEFARAD, vol. 81:1, enero-junio 2021, págs. 21-42. ISSN: 0037-0894. https://doi.org/10.3989/sefarad.021-002 


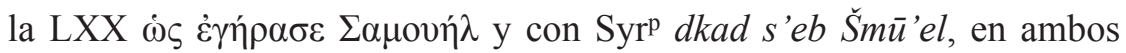

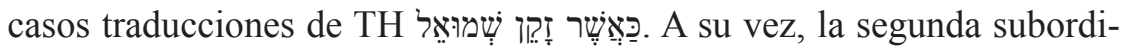
nada, esta de relativo, 'qym bnwy d'ynyn l'ysr'yl ('designó a sus hijos

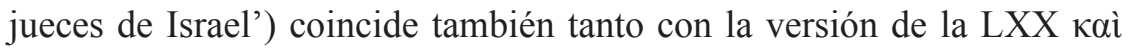

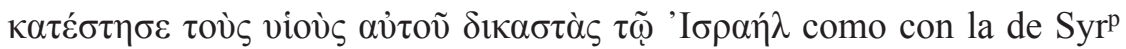
'bad lbnawhy dayone 'al Isra'el, vertiendo ambas el TH

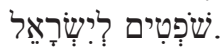

A priori pudiera tratarse de una traducción realizada a partir de cualquiera de los dos textos, sin embargo, como en los casos precedentes, creemos que la traducción del texto ACP ha sido hecha a partir de la LXX, aunque cotejada con otra versión, que en este caso pudiera ser la de Syrp. Aludimos a esta doble posibilidad porque, si nos fijamos, ACP omite la conjunción copulativa $w$ - ('y'), con valor de relativo, delante de la forma aphel 'aqìm, al igual que sucede con $\mathrm{Syr}^{\mathrm{p}}$, y algunos manuscritos de la $\mathrm{LXX}^{41}$. No deja de tener interés para la tradición textual como Syr ${ }^{\mathrm{p}}$ vierte

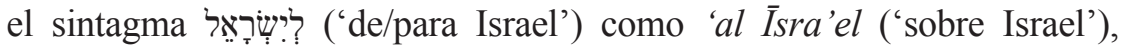
cuando ACP lee l'ysr'yl ('de/para Israel'), interpretando perfectamente de este modo, con la preposición $l$ - ('a, para'), la función dativa que desempeña el sintagma. Otro aspecto interesante para justificar que LXX representa el texto base utilizado para la traducción es que la forma aph. 'aqìm representa el significado básico de $\kappa \alpha \theta i ́ \sigma \tau \eta \mu \imath$ ('poner, designar') ${ }^{42}$ y no la del peal 'bad ('hacer'), que en $\kappa a \theta i ́ \sigma \tau \eta \mu$ es secundaria.

\section{8,2}

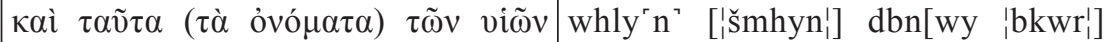

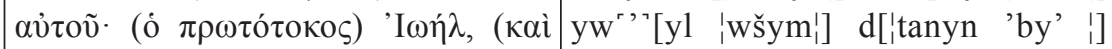

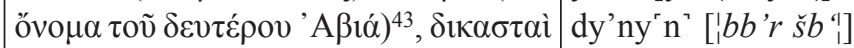

( $\dot{\varepsilon} \nu \mathrm{B} \eta \rho \sigma \alpha \beta \varepsilon \varepsilon \dot{\varepsilon})$

Y estos (son los nombres) de sus hijos: (el primogénito) Joel (y el nombre del segundo, Abiyá), jueces (en Bersabé)

${ }^{41}$ The Old Testament in Greek II/1, ed. Brook, McLean, Thackeray, pág. 23 ad locum.

${ }^{42} C f$. Vasile BавотA, The Institution of the Hasmonean High Priesthood (LeidenBoston: Brill, 2014) pág. 90.

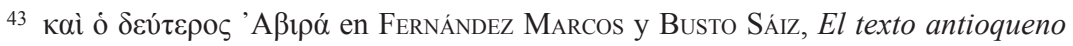
de la biblia griega I, pág. 21 ad locum. 
En este segundo versículo del capítulo octavo volvemos a tener un texto fragmentario con las siguientes dos correspondencias de interés: en la primera tenemos que ACP whlyn ('y estos') es traducción de LXX кà̀ $\tau \alpha \tilde{v} \tau \alpha$ ('y estos') y no de Syrp wahwō ('y era'), referido a šem ('nombre'), cf. TH וַיהּי שֶׁם ('y era el nombre'); y otro tanto sucede con el sintagma

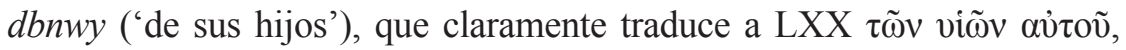
pero no a Syr" breh ('de su hijo' < TH רִִּ ). Las dos correspondencias

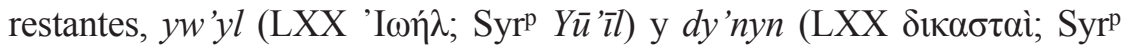
dayonē) no ofrecen información de interés para el presente caso de estudio.

De acuerdo con la dependencia plena que exhibe ACP en este versículo con la versión que ofrece la LXX, creemos que quedan justificadas las cuatro restituciones que hemos incluido en la edición: en primer

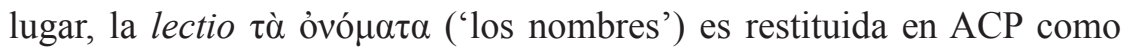
šmhyn. Del mismo modo, podemos restituir la lectura $\pi \rho \omega \tau$ б́toко ('primogénito') con $b k w r$, atestiguada por lo demás en otro fragmento, casualmente correspondiente a 1 Sam 6,744. En tercer lugar, el texto de

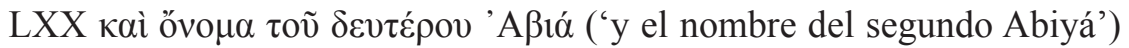
nos permite, asimismo, restituir con una altísima probabilidad el texto fragmentario en ACP como wšym dtnyn 'by' ( $c f$. Syr ${ }^{\mathrm{p}}$ wšem tenyoneh

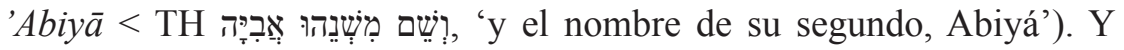
finalmente, la cuarta restitución está representada por la lectio غ̇v

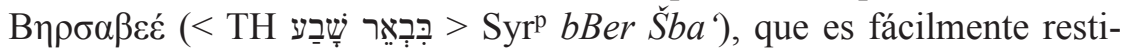
tuible como $b b^{\prime} r s^{\prime} b$; , en este caso con la ayuda de Syrp.

\section{8,3}

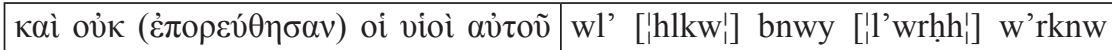

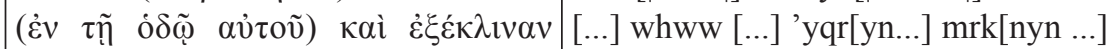

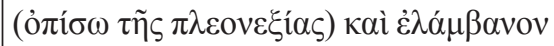

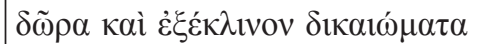

Y no (anduvieron) sus hijos (por su camino) y se desviaron (tras la ganancia) y aceptaron (regalos y) se desviaron de los juicios rectos

Este tercer versículo, asimismo fragmentario, presenta una serie de seis correspondencias que enumeramos a continuación: wl' ('y no'),

${ }^{44}$ Cf. A Corpus of Christian Palestinian Aramaic. Volume I, ed. Müller-Kessler y SoKoloff, pág. 93 col. b.

SEFARAD, vol. 81:1, enero-junio 2021, págs. 21-42. ISSN: 0037-0894. https://doi.org/10.3989/sefarad.021-002 


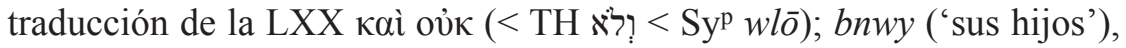
que lo es de oi vioì av̉

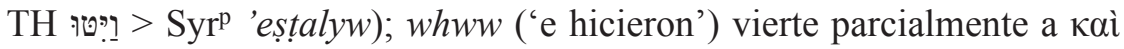

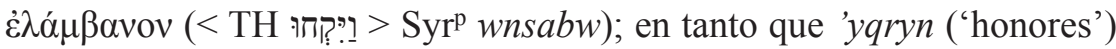
vierte a $\delta \tilde{\omega} \rho \alpha\left(<\mathrm{TH}\right.$ ש $>$ Syrp $^{\mathrm{p}}$ šūhdō) y por último mrknyn ('se incli-

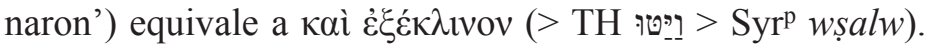

En el plano de las posibles restituciones tenemos la correspondiente al primer vacat, cuya lectura en la LXX غ̇ंo nes de Syr ${ }^{p}$, Syr ${ }^{\mathrm{je}}$ y B $\mathrm{B}^{\mathrm{s}}$ (halekw), nos permiten restituirla como hlkw ('[ellos]

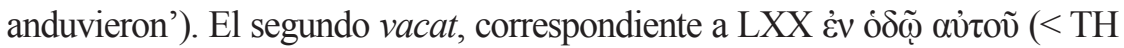

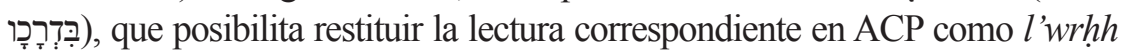
('por su camino'), sintagma preposicional que se encuentra atestiguado en otro fragmento que ofrece 1 Sam $1,18^{45}$. Syr ${ }^{\mathrm{je}}$ y B $\mathrm{B}^{\mathrm{s}}$ leen $b^{\prime}$ urrheh (cf. Syr ${ }^{\mathrm{p}} b^{\prime}$ ürhoteh), que también sería posible como restitución, si bien preferimos la lectio anterior por estar documentada en ACP y en concreto en el libro de Samuel.

B) Frag. XVII $(8,4 a)-$ Frag. XVII ${ }^{\mathrm{r}}$

$$
8,4
$$

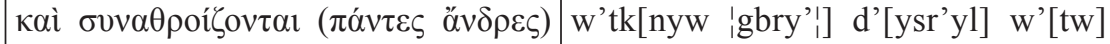

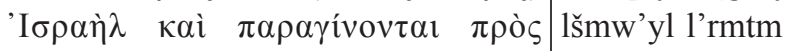

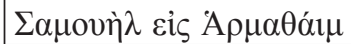

Y se congregaron (todos los varones) de Israel y fueron a Armataim ante Samuel

Este versículo cuarto está prácticamente completo, con la excepción de un término, que como veremos es susceptible de restitución en ACP. Las correspondencias las podemos establecer en las tres secuencias si-

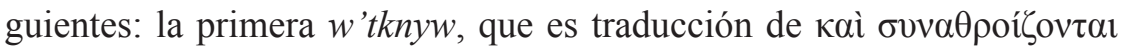

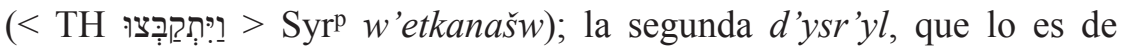

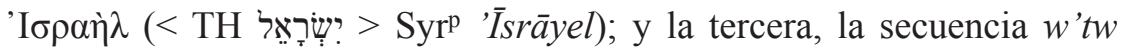
lšmw'yl l'rmtm ('y fueron hasta Samuel, en Armataim'), que vierte a kaì

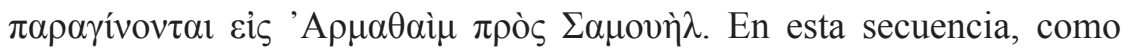
podemos ver, el orden de palabras que exhibe ACP coincide con la lec-

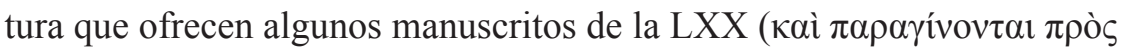

${ }^{45}$ Cf. A Corpus of Christian Palestinian Aramaic. Volume I, ed. MüLler-Kessler y SoKoloff, pág. 88 col. b. 


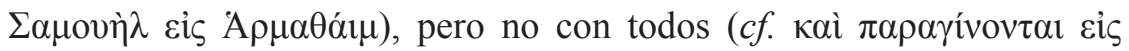

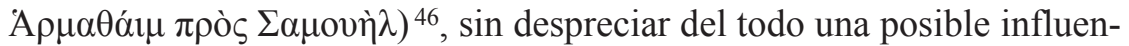

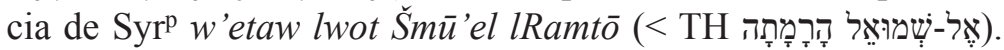

Como hemos indicado, la restitución del único término que falta en este versículo es susceptible de ser, cuando menos, planeada. La lectio que ofrece la LXX es el plural ó $v \delta \rho \varepsilon \varsigma$ ('hombres [adultos]'), cuyo equivalente en ACP creemos que puede ser bien gbr' o 'nwšy'. Cabría la posibilidad de que fuese sbyn ('ancianos', $c f$. Syr ${ }^{\mathrm{p}}$ kulhūn sbè dabnay 'İsra'el, i.e. 'to-

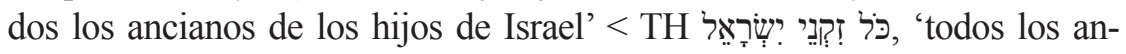
cianos de Israel'), pero esta lectura la tenemos atestiguada como traducción de $\pi \rho \varepsilon \sigma \beta \hat{t} \tau \varepsilon \rho o r\left(1\right.$ Sam 4,3) en uno de los fragmentos conservados ${ }^{47}$. En

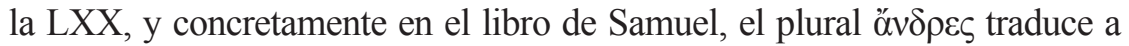

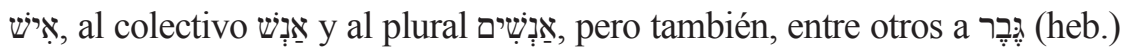
y גָּ (aram.) ${ }^{48}$. De hecho, en uno de los fragmentos de ACP gbry' es utilizado para verter a ò $v \delta \rho \tilde{\omega} v(1 \mathrm{Sam} 4,2)^{49}$, lo que nos permite suponer que el plural gbry' parezca ser la lectio más apropiada para la restitución.

\section{8,5}

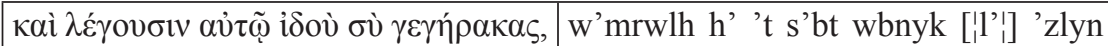

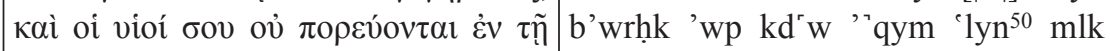

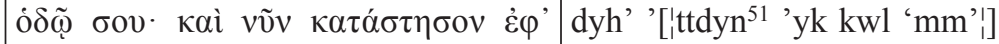
$\dot{\eta} \mu \tilde{\alpha} \varsigma \beta \alpha \sigma \imath \lambda \varepsilon ́ \alpha(\delta ı \kappa \alpha ́ \zeta \varepsilon ı v ~ \dot{\eta} \mu \tilde{\alpha} \varsigma, \kappa \alpha \theta \dot{\omega} \varsigma$

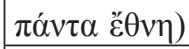

y le dijeron: «he aquí que eres viejo y tus hijos no andan por tu camino; ahora desígnanos un rey que (nos juzgue como a las demás gentes)»

46 Fernández Marcos y Busto Sáiz, El texto antioqueno de la biblia griega I, pág. 21 ad locum. Cf. The Old Testament in Greek II/1, ed. Brook, McLean, Thackeray, pág. 23 ad locum.

47 Cf. A Corpus of Christian Palestinian Aramaic. Volume I, ed. Müller-Kessler y SoKoloff, pág. 92 col. a.

48 Cf. Takamitsu Muraoka, A Greek $\approx$ Hebrew/Aramaic Two-way Index to the Septuagint (Louvain-Paris-Walpole, MA: Peeters, 2010) pág. 11a.

49 Cf. A Corpus of Christian Palestinian Aramaic. Volume I, ed. Müller-Kessler y SoKoloff, pág. 92 col. a.

${ }^{50}$ Ms. sic pro 'lynn, cf. A Corpus of Christian Palestinian Aramaic. Volume I, ed. Müller-Kessler y Sokoloff, pág. 98 (n. 1).

51 GolteIn sugiere [d]'[yn ...], cf. A Corpus of Christian Palestinian Aramaic. Volume I, ed. MüLler-Kessler y SoKoloff, pág. 98 (n. 2). 
En este caso nos falta la parte final del versículo, que lamentablemente no se nos ha conservado, con un vacat, además, en la segunda oración coordinada copulativa, que es de fácil restitución, ya que se trata del adverbio negativo $l$ ' ('no' < LXX oủ < TH לא > Syr $l \bar{l}$ ), que restituimos convenientemente en la edición. El texto conservado en este versículo representa una traducción literal del que exhibe la $\mathrm{LXX}^{52}$, aunque ACP ofrece una adición, la conjunción 'wp ('también'), ausente en LXX, Syr ${ }^{p}, \mathrm{TH}$ y Vg. Una segunda restitución es la del vacat final, que creemos puede ser restituida como ttdyn 'yk kwl 'mm' ('y nos ad-

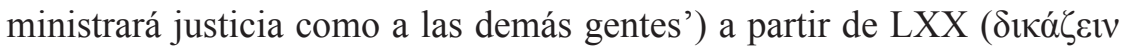

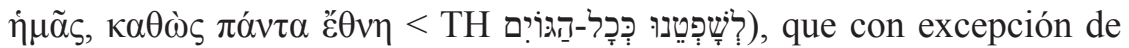
la forma ethtaphel 'ttdyn es la lectura que ofrecen Syr ${ }^{\mathrm{p}}$ y Syrie (dndünan ayk kulhūn 'ammē).

$$
8,6
$$

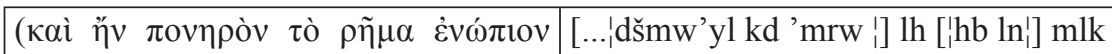

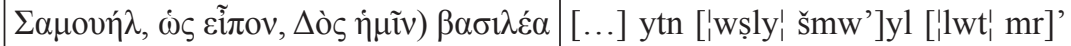

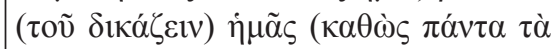

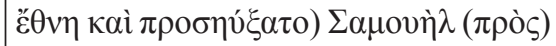
Kúprov

(Y mala fue la palabra a ojos de Samuel cuando dijeron: «danos) un rey (para que) nos (juzgue». Y oró) Samuel (al) Señor

Lamentablemente, este versículo se encuentra muy fragmentado y ello no nos permite poder efectuar restituciones sin correr más riesgo del debido. Con todo, la técnica de traducción literal utilizada por el traductor posibilita que podamos completar algunos vacat del versículo. Es el caso del imperativo peal + preposición y pronombre enclítico de $1^{\text {a }}$ p. pl. $h b \ln$ ('danos'), que creemos debe figurar delante del sustantivo

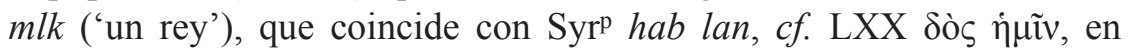

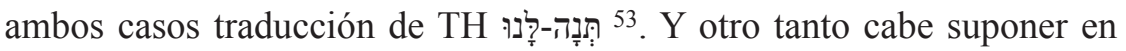
el vacat que tenemos delante de la lectura šmw'yl ('Samuel'), donde

${ }^{52}$ La secuencia de ACP w'mrwlh h' 't s'bt wbnyk l' se corresponde plenamente con la lectura que ofrece Syrp.

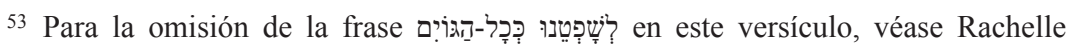
GILmour, Representing the Past: A Literary Analysis of Narrative Historiography in the 
cabe suponer el pael wṣly ('y oró'), propuesta fundada en otro fragmento donde figura wṣly ( $c f$. Syr ${ }^{\mathrm{p}}$ wṣali < TH LXX $\pi \rho \circ \sigma \eta v ́ \xi \alpha \tau o$ en 1 Sam 1,10 ${ }^{54}$. Asimismo, el vacat que figura entre las lectiones šmw'yl ('Samuel') y $m r$ ' ('el Señor') cabe ser restituido con la preposición $l w t$ ('a, ante'), lectura atestiguada en el mismo fragmento al que acabamos de referirnos que contiene 1 Sam 1,10 (wṣlyt lwt mr',

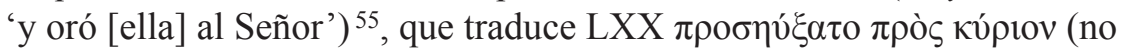

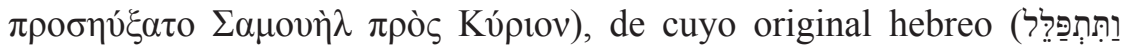

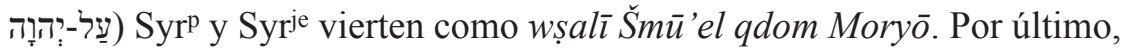
el primer vacat, pese a que también tiene una extensión considerable en este versículo, sin embargo podemos restituir la parte final del mismo

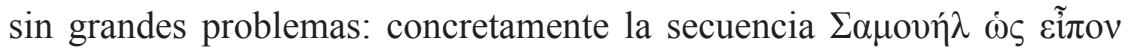

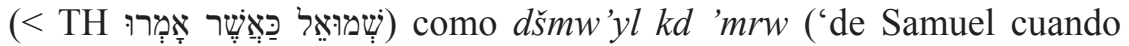
dijeron'), idéntica lectura a la que dan $\operatorname{Syr}^{\mathrm{p}}$ y Syr ${ }^{\mathrm{je}}$ (dšmū'el kad 'emarw).

8,7

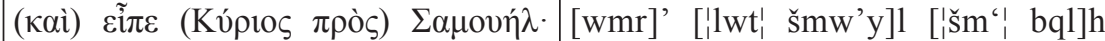

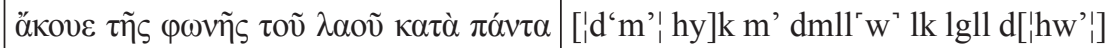

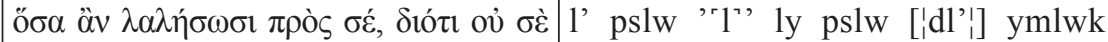

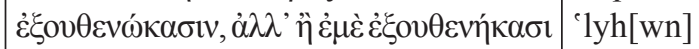

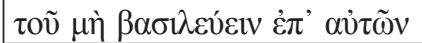

(Y) dijo (el Señor a) Samuel: «(oye) la voz (del pueblo) según todo lo que te han hablado, porque no es a ti a quien han rechazado, sino que a mí han rechazado (para no) reinar sobre ellos

Este versículo nos ofrece interesantes posibilidades de restituir lecturas en los vacat que presenta el texto. La versión literal que exhibe ACP nos facilita enormemente la labor. Así, en el primer caso, es fácil inferir que se trata de la conjunción copulativa $w$-, tal como restituimos en la edición. El segundo vacat creemos que hay que restituirlo con la prepo-

Book of Samuel (=Supplements to Vetus Testamentum 143 [Leiden-Boston: Brill, 2011]) págs. 175, 195 y 197.

54 Cf. A Corpus of Christian Palestinian Aramaic. Volume I, ed. Müller-Kessler y SoKoloff, pág. 87 col. a.

55 Cf. A Corpus of Christian Palestinian Aramaic. Volume I, ed. Müller-Kessler y SoKoloff, pág. 87 col. a.

SEFARAD, vol. 81:1, enero-junio 2021, págs. 21-42. ISSN: 0037-0894. https://doi.org/10.3989/sefarad.021-002 
sición $l w t$, como sucede en el caso anterior, donde también corresponde

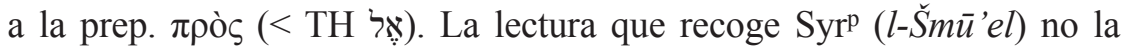
creemos posible, porque de ser tal debería ir unida al nombre propio al que antecede, situación que obviamente no se da en ACP. El tercer y cuarto vacats, por su parte, son restituibles respectivamente con el imperativo peal $\check{s} m$ ' ('oye') y el sintagma $d$ ' $m$ ' ('de la gente/pueblo'), siendo la lectura šm ' bqlh d'm' ('oye la voz del pueblo') la traducción de LXX

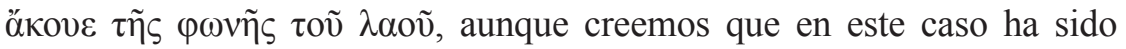
influenciada por Syr ${ }^{\mathrm{p}}$ šma 'bqoleh d'amō, ambas vertidas a partir de $\mathrm{TH}$

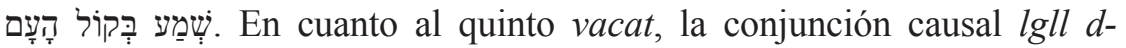
('porque') ${ }^{56}$ exige la lectio $h w$ ', documentada en un fragmento ACP del NT $(\text { Jn } 2,25)^{57}$. Finalmente, el sexto vacat, correspondiente al par $\tau$ oṽ $\mu \grave{\eta}$ es restituible por $d l$ ' ('para que no'), gracias a la lectura que proporciona Syr ${ }^{\mathrm{p}}$ : $d l \bar{o}$, que tanto esta como la de la LXX vierte a TH כִּי לא.

8,8

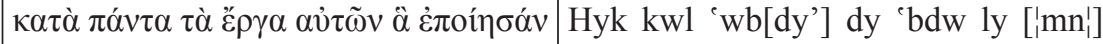

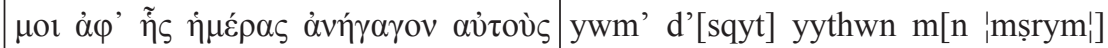

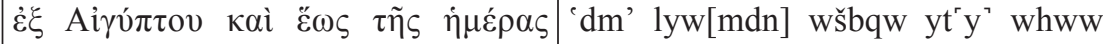

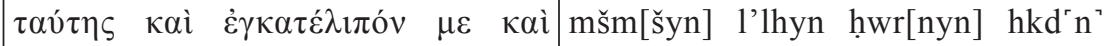

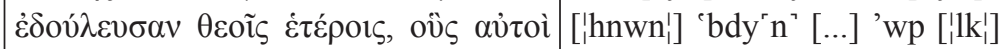

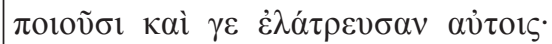

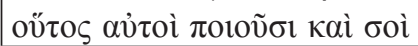

Según todos los hechos que me hicieron (desde) el día que les saqué de (Egipto) hasta este día, me abandonaron y sirvieron a otros dioses; así hacen ellos ahora contigo

Como en los casos anteriores, también en este nos encontramos ante una traducción verbum e verbo, hecho que nos posibilita realizar restituciones en el texto de ACP. De este modo, el primer vacat es fácilmente restituible con la preposición $m n\left(<\mathrm{LXX} \alpha \dot{\alpha} \varphi^{\prime}<\mathrm{TH}\right.$ ’̣), lectura atestiguada por $\mathrm{Syr}^{\mathrm{p}}$ y por Syr ${ }^{\mathrm{je}}$ (men). El segundo vacat es, asimismo, fácil

56 Müller-Kessler, Grammatik des Christlich-Palästinisch-Aramäischen, pág. 149.

57 Cf. A Corpus of Christian Palestinian Aramaic. Volume IIA. The Christian Palestinin Aramaic New Testament Version from the Early Period Gospels, ed. Christa MüLLERKessler y Michael SoKoloff (Groningen: Styx Publications, 1998) pág. 195 col. a. 
de restituir con la lectura mṣrym ('Egipto'), gracias al literalismo de la

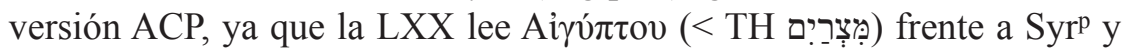
Syr je, que leen 'ar'ō $d$-Meșreyn ('la tierra/el país de Egipto'). El tercer vacat es restituible, también con la ayuda de LXX, como hnwn ('ellos'

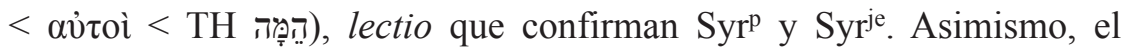
quinto vacat es también fácil de restituir, porque además de la lectura

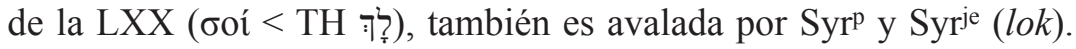

\section{Conclusiones}

El análisis precedente nos permite corroborar que, como sucedía con la versión del fragmento del $G n$ estudiado con anterioridad ${ }^{58}$, el traductor de los fragmentos ACP -siguiendo una estrategia utilizada por otros autores siriacos que se sirvieron de varias versiones para sus traducciones o comentarios ${ }^{59}$ - ha utilizado como texto base de su versión un texto griego de la LXX, que vertió siguiendo la técnica verbum e verbo; pero además del texto griego, como hemos podido comprobar, también se ha servido de una o más versiones siriacas, una de la cuales todo parece indicar que sea la versión representada por la Peshīttā. No perdamos de vista, en este sentido, que las escuelas de traductores siriacos, y arameos en general, fueron en cierto grado el resultado de una cultura bilingüe en la que la lengua fuente tenía un decisivo prestigio ${ }^{60}$.

Además, el traductor de los fragmentos ACP no solo recurrió a un texto griego de la LXX, pues como hemos visto este texto fue cotejado con otra $\mathrm{u}$ otras versiones siriacas, con dos objetivos esenciales: por un lado precisar, lexicalmente, el término más adecuado en ACP a partir de la opción siriaca ( $c f$. comentario en vs. 7,15.16.17 ...); y por otro lado, si bien solo en contadas ocasiones, cambiar el orden de las palabras que

\footnotetext{
58 Monferrer-SALA, «Dos fragmentos en arameo cristiano-palestinense del libro del Génesis».

59 Un caso interesante es el que presenta el 'Comentario al Génesis' de Eusebio de Émesa: R.B. Ter Haar Romeny, A Syrian in Greek Dress: The Use of Greek, Hebrew, and Syriac Biblical Texts in Eusebius of Emesa's Commentary on Genesis (=Traditio Exegetica Graeca 6 [Louvain: Peeters, 1997]) págs. 34-88.
}

60 BRock, «Aspects of Translation Technique in Antiquity», pág. 74. 
presenta el texto griego ( $c f$. comentario en vs. 7,17 y 8,4). Además, junto a estas opciones el traductor también recurrió a estrategias de traducción propias, fundamentalmente la técnica de la modulación en su doble vertiente, amplificativa y reductiva, que en ocasiones conlleva un cambio exegético, o bien acomoda la traducción al contexto de la narración e incluso clarifica una lectura o una expresión.

La importancia de cuanto acabamos de concluir no es en modo alguno baladí. En la presente investigación, si bien revestía una evidente relevancia, no era nuestra única intención confirmar la hipótesis anterior de la utilización de varias versiones en un nuevo fragmento ACP. Además de corroborar esa hipótesis, en función de una serie de evidencias obtenidas en el análisis de la traducción y con un criterio metodológico fundamentado en aquellas, pretendíamos poder llevar a cabo la restitución de toda una serie de lecturas ilegibles que exhibe el fragmento, que las ediciones de Goshen-Gottstein y de Müller-Kessler y Sokoloff no ofrecen.

El criterio metodológico adoptado se basa en una doble evidencia: en primer lugar, en el literalismo que, salvo muy contadas excepciones, exhibe la versión ACP con respecto al texto griego; en segundo lugar, los testigos léxicos y sintácticos que ofrecen las versiones siriacas de la Peshīttāa, y la utilizada por Jacobo de Edesa, con las que poder avalar, en su caso, las restituciones que planteamos. Y súmese a lo anterior, lo que podemos calificar como 'evidencias léxicas internas', esto es, la confirmación de términos o sintagmas que exhiben otros fragmentos pertenecientes al corpus de ACP.

Los dos objetivos que justifican este trabajo, el análisis de la versión por un lado y la restitución de lecturas ilegibles por otro, nos permiten conocer más en detalle cuál fuera el proceso de traducción seguido por el traductor, al tiempo que poder restituir las lecturas ilegibles del fragmento estudiado con una metodología apropiada. En consecuencia, es nuestra intención que el criterio metodológico utilizado en este trabajo pueda ser utilizado en adelante como herramienta en un proceso integral de restitución de las lecturas ilegibles que exhibe el resto de fragmentos bíblicos conservados en el corpus ACP.

Recibido: 06/01/2021

Aceptado: 14/03/2021 